\title{
s Supplementary Information
}

Table S1 Range of hydrological parameters of GR4J model

\begin{tabular}{llll}
\hline Parameter & description & L & U \\
\hline X1 & production store capacity [mm] & 200 & 300 \\
X2 & Inter-catchment exchange coefficient [mm/d] & 0 & 5 \\
X3 & routing store capacity [mm] & 10 & 80 \\
X4 & unit hydrograph time constant [d] & 0 & 5 \\
\hline
\end{tabular}



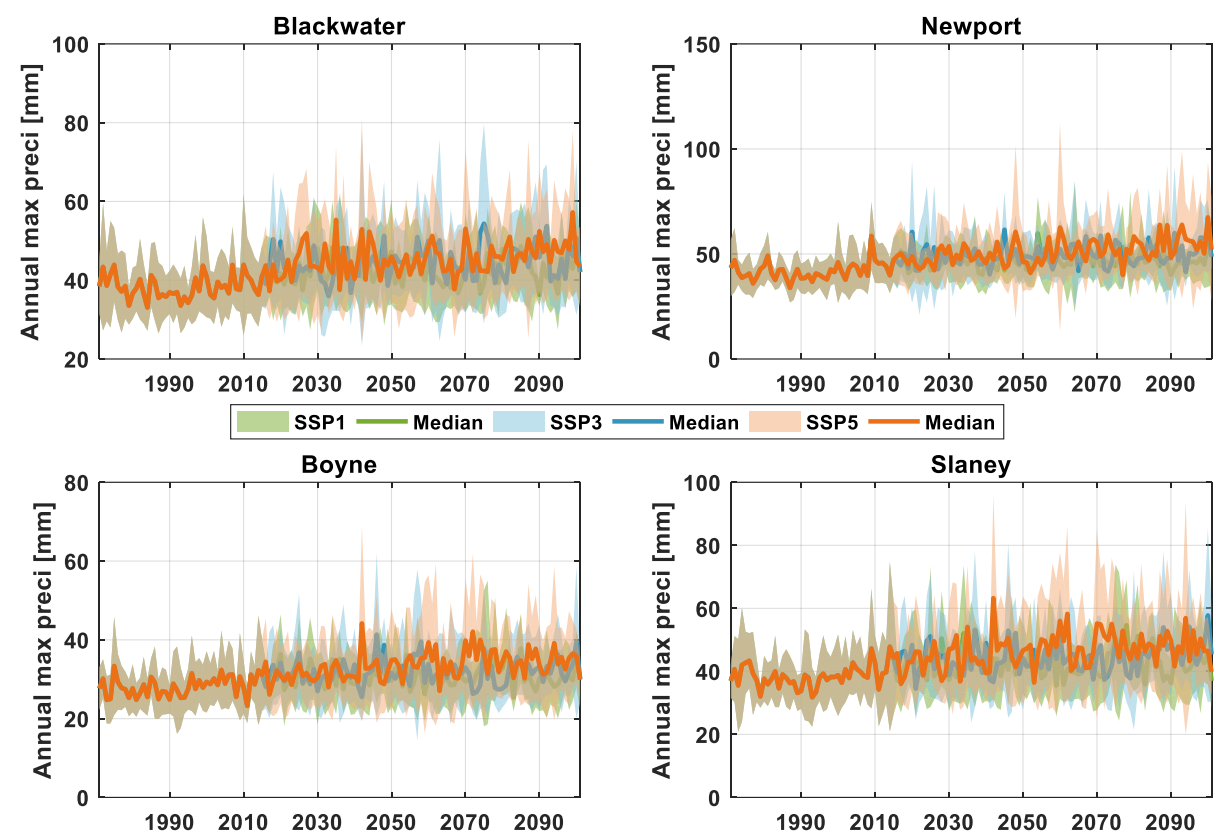

15 Figure S2. Projected annual maximum precipitation as simulated by 12 CMIP6 GCMs forced with SSP1, SSP3 and SSP5 for each catchment for the period 1976-2100. The orange, blue and green shaded areas represent the model ensemble spread (95\% confidence interval) for SSP5, SSP3 and SSP1, respectively. The solid lines represent the ensemble median for each SSP scenario. 

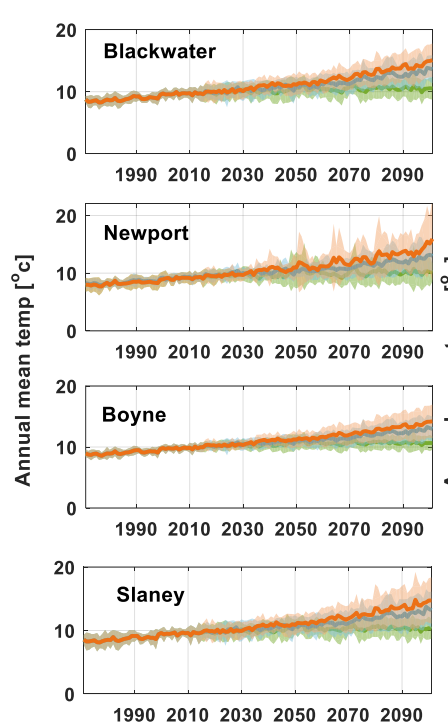
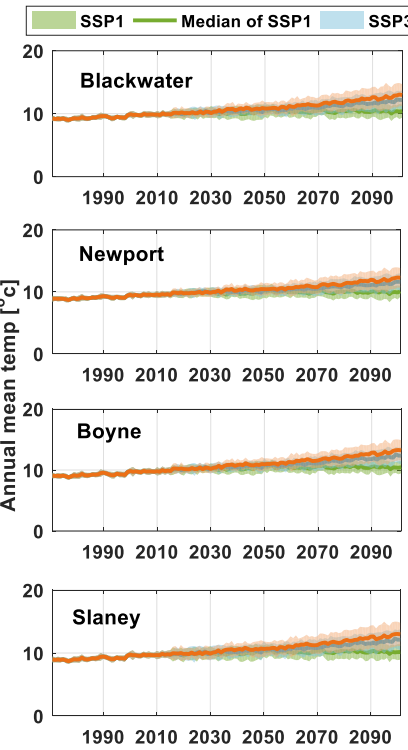

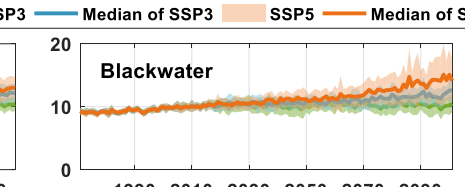

199020102030205020702090
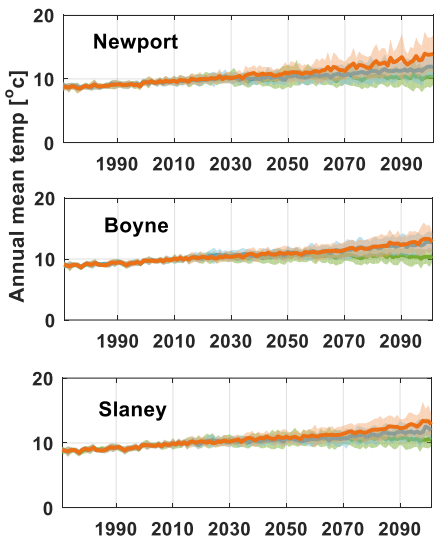
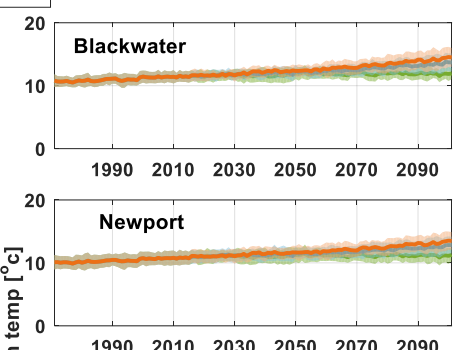


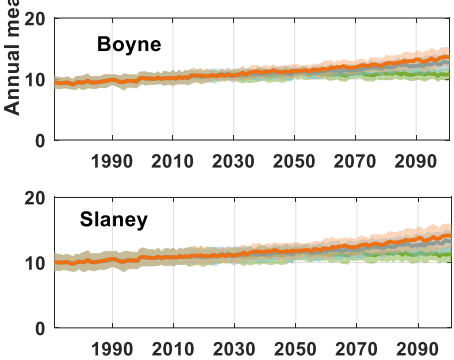

Figure S3. Comparison of temperature bias correction using BSM (first column from left), CF (second column from left), 30 EQM (third column from left) and the fourth column is uncorrected air temperature for each catchment. 


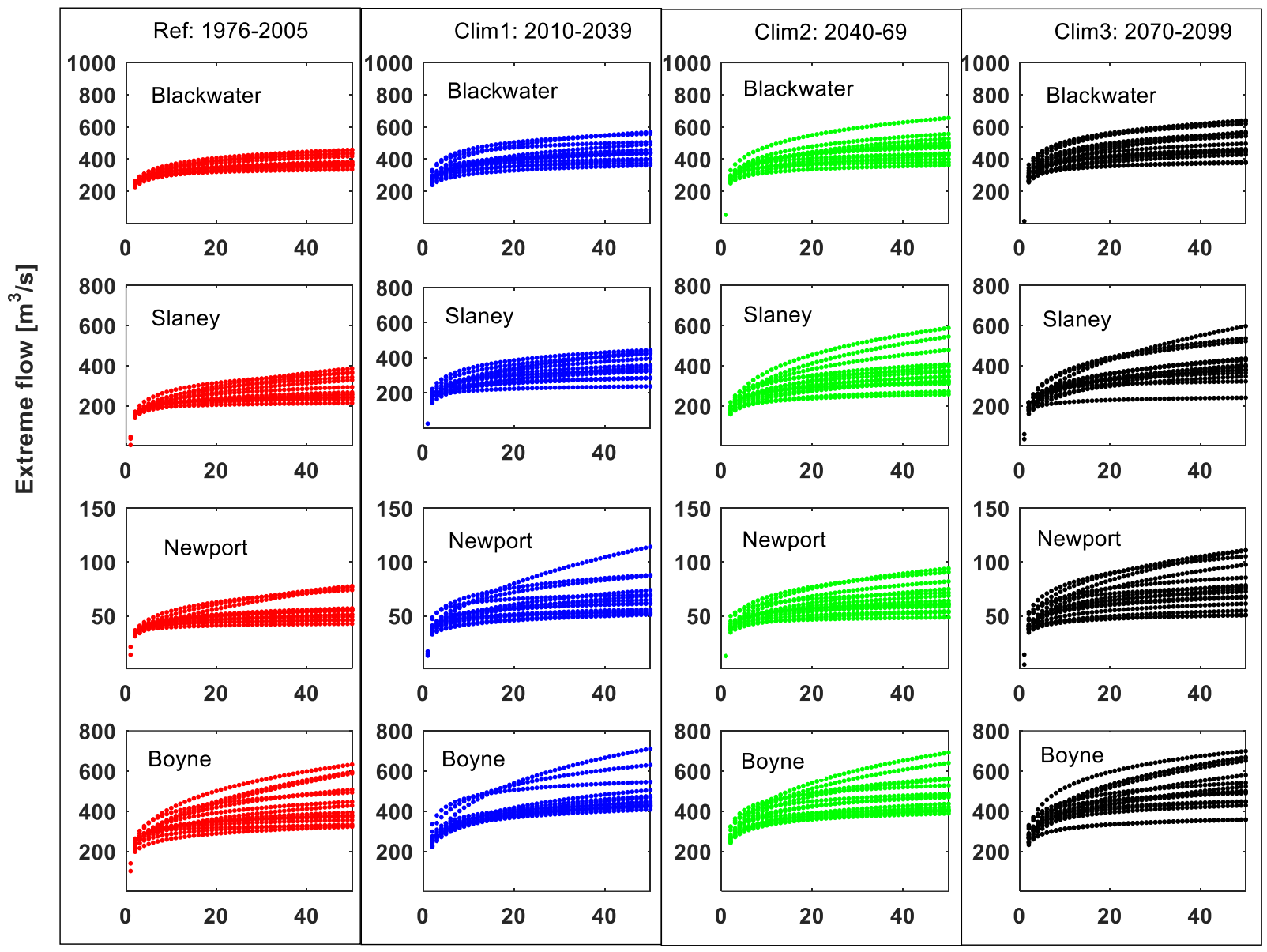

40

Return period [year]

Figure S4. Flood frequency curves at different return periods for the reference period (1976-2005), the 2020s (2010-2039), the 2050s (2040-2069) and the 2080s (2070-2099) in each of the four study catchments. Each colour represents each climate period, red for reference period, blue very near future, green near future and black far future. Each line in each colour represent single climate model simulated from the best hydrological parameter set and DGQM bias correction. 


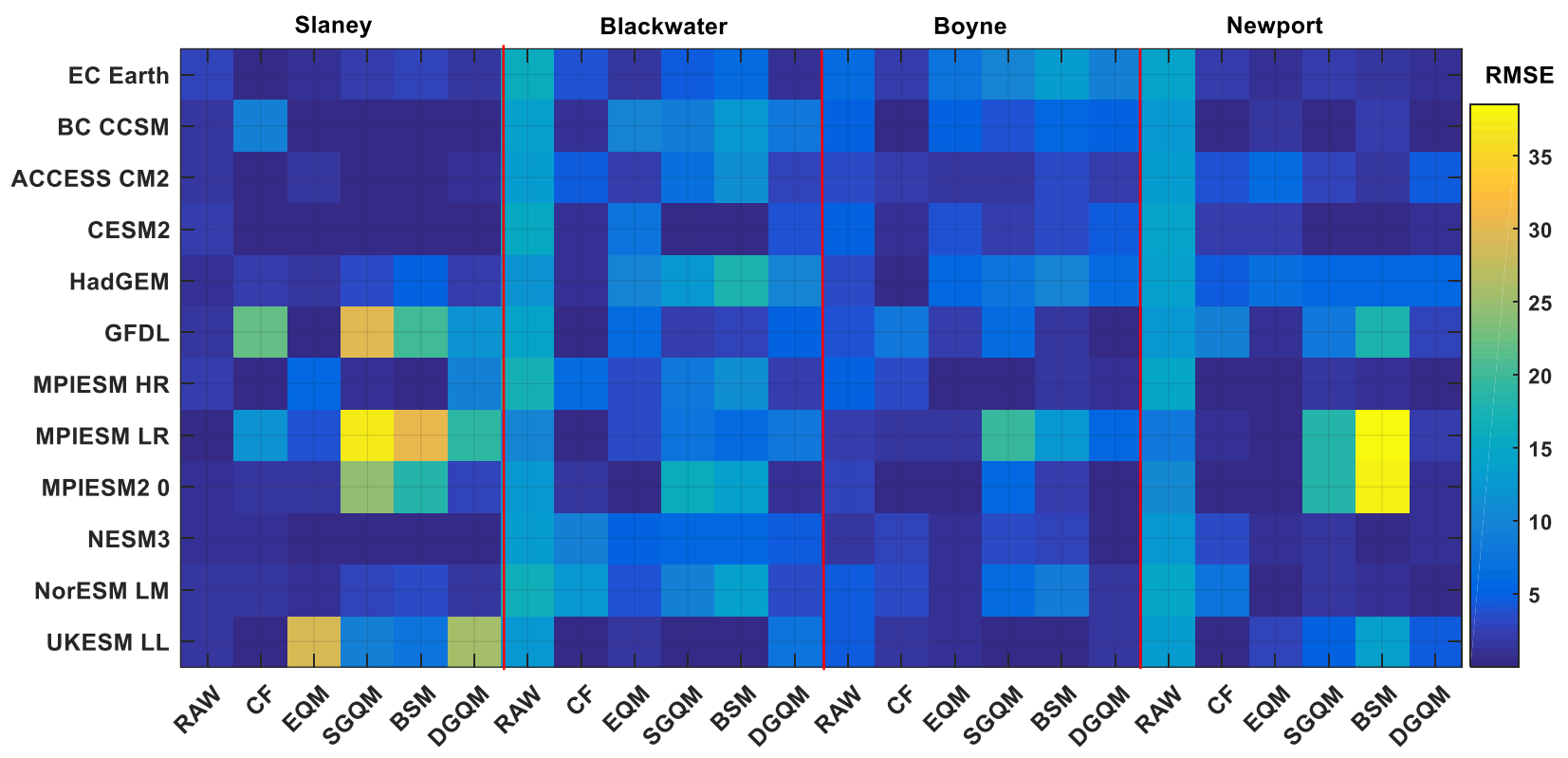

Figure S1. Root Mean Square Error (RMSE) of corrected annual maximum precipitation using five bias correction methods applied to 12 CMIP6 GCMs. The y-axis indicates each climate model and x-axis represents each climate bias correction method. 